\title{
Nuclear magnetic resonance spectrum of ${ }^{31} P$ donors in silicon quantum computer
}

\author{
A.A. Larionov, L.E. Fedichkin, A.A. Kokin, K.A. Valiev \\ Institute of Physics and Technology, Russian Academy of Sciences, 34, Nakhimosky pr., Moscow, \\ 117218, Russia \\ e-mail: qubit@ftian.oivta.ru
}

\begin{abstract}
The influence of the electric field created by a gate potential of the silicon quantum computer on the hyperfine interaction constant (HIC) is obtained. The errors due to technological inaccuracy of location of donor atoms under a gate are evaluated. The energy spectra of electron-nuclear spin system of two interacting donor atoms with various values of $\mathrm{HIC}$ are calculated. The presence of two pairs of anticrossing levels in the ground electronic state is shown. Parameters of the structure at which errors rate can be greatly minimized are found.
\end{abstract}

\section{INTRODUCTION}

The first approach to the NMR quantum computer realization simultaneously proposed in 1997 by two groups of investigators [1,2], and then experimentally confirmed [3, 4 , consists in using organic liquids, where different molecules with a few connected with each other nonequivalent nuclear spins-qubits function as independent quantum computers. Another approach, which is qualitatively different from the bulk-ensemble one and is still unrealized, was proposed and analyzed in details by Kane [5] and elaborated in [8, 13, 14]. It is based on creating artificial multi-spin system and using individual addressing to different spins-qubits. For this purpose it is suggested to use silicon-based structure of MOS-type, where donor atoms of stable phosphorus isotope ${ }^{31} \mathrm{P}$ are implanted into the thin layer of spinfree silicon isotope ${ }^{28} \mathrm{Si}$ at a definite depth. Donor atoms replace silicon ones in knots of crystal lattice. Such a donor has shallow impurity states, which have a large magnitude of the effective Bohr 
radius and nuclear spin $I=1 / 2$. Every donor atom with nuclear spin in semiconductor structure is supposed to be placed regularly with adequate accuracy under its "own" control metallic gate (gate A), which is separated from the silicon surface by a thin dielectric layer (for example, silicon oxide with the thickness of about some nanometers). A-gates form linear regular structure of arbitrarily length with period l (Fig. 1). Changing of the electric potential of J-gates, which are placed between A-gates, allows us by redistributing electron density between neighboring donors to control the degree of overlapping of electron wave functions, which are localized on neighboring donors a and b, and to control the constant of exchanging interaction $J$, and the constant of scalar interaction of their nuclear spins $I_{a b}$ as well. It is assumed that with the help of the electric field, induced by A-gates, one can change the distribution of electronic density near the nuclei in the ground state by choosing individually, correspondingly, each donor atom nuclear spin resonance frequency, which is determined by the hyperfine interaction with its electron spin. This allows to fulfill quantum operations by the way of selective reaction of resonance radio-frequency pulses on nuclear spins of the given donors. The second section of the work studies the influence of the electric field, created by potential V on the A-gate, on hyperfine interaction constant (HIC) of the donor atom in two cases: for gates having the form of a round disc and that of a strip. In the third section HIC fault approximation is made, which is determined by the technological placement inaccuracy of donor atom under the gate. In the fourth section the results of full calculation of the electron-nuclear spin system energy spectrum of two interacting donor atoms with different magnitude of HIC are demonstrated. Separately the phenomenon of hyperfine energy levels crossing and anti-crossing is investigated, owing to which the information about nuclear spin state is transferred to the electron spin of donor atom when moving through the point of crossing. This property was suggested to be used in the process of electrical measurement of a definite nuclear spin state [0,11]. It is also very important when using magnetically-mechanic resonance method [12]. 


\section{DEPENDENCE OF THE HYPERFINE INTERACTION CONSTANT ON THE ELECTRICAL FIELD}

Spin Hamiltonian of the hyperfine interaction is:

$$
\hat{H}_{I S}=A(\hat{\vec{I}} \hat{\vec{S}}), A(V)=\frac{8 \pi}{3}\left|\Psi_{0}(0, V)\right|^{2} 2 \mu_{B} g_{N} \mu_{N} \cdot\left(\frac{\mu_{0}}{4 \pi}\right)
$$

where $\mu_{N}=5.05 \cdot 10^{-27} \mathrm{~J} / \mathrm{T}$ - nuclear magneton, $\mu_{0}=4 \pi \cdot 10^{-1} \mathrm{~T}^{2} \mathrm{sm}^{3} / J, g_{N}=2,26$

— Lande's factor for ${ }^{31} P, \mu_{B}=9.27 \cdot 10^{-24} \mathrm{~J} / \mathrm{T}$ - Bohr magneton, and $\Psi_{0}(0, V)$ - wave function of the electron in the ground state placed on the nuclei. Experiments give the value of the probability density of the electron to be on the nuclei $\left(\Psi_{0}(0)\right)^{2}=0.43 \cdot 10^{24} \mathrm{sm}^{-3}$ at $\mathrm{V}=0$ [6].

Taking into account that intrinsic semiconductor at low temperatures behaves like dielectric and supposing that $D>>l_{A} \sim c>>d$ (Fig. 1), we express the electric field on the donor atom depending on potential $\mathrm{V}$ on gate $\mathrm{A}$, (round disc with radius $a=\frac{l_{A}}{2}$ ), which lies on the surface of the dielectric. To find the electric potential induced by the round disc at the point with the coordinates $\vec{r}^{\prime}=\left(\rho^{\prime}, z^{\prime}\right), \rho^{\prime 2}=x^{\prime 2}+y^{\prime 2}$, we use known expression [7]:

$$
\varphi\left(\rho^{\prime}, z^{\prime}\right)=\frac{2 V}{\pi} \operatorname{arctg} \sqrt{\frac{2 a^{2}}{\rho^{\prime 2}+z^{\prime 2}-a^{2}+\sqrt{\left(\rho^{\prime 2}+z^{\prime 2}-a^{2}\right)^{2}+4 a^{2} z^{\prime 2}}}}
$$

On the line, which passes through the gate center $\rho^{\prime}=\sqrt{x^{\prime 2}+y^{\prime 2}}=0$, near the donor atom $\left(z^{\prime}=c\right)$, we receive:

$$
\varphi\left(\rho^{\prime}, z^{\prime}\right)=\frac{2 V}{\pi} \operatorname{arctg} \frac{a}{c}-E_{c}\left(z^{\prime}-c\right)+\frac{E_{c}^{\prime}}{2}\left(z^{\prime}-c\right)^{2}-\frac{E_{c}^{\prime \prime}}{2} \rho^{\prime 2}+\ldots,
$$

where

$$
E_{c}=\frac{2 V}{\pi} \cdot \frac{a}{a^{2}+c^{2}}, E_{c}^{\prime}=\frac{4 V}{\pi} \cdot \frac{a c}{\left(a^{2}+c^{2}\right)^{2}}, E_{c}^{\prime \prime}=\frac{4 V}{\pi} \sqrt{2} \frac{a c^{4}}{\left(a^{2}+c^{2}\right)^{7 / 2}}
$$

the electric field, its gradient in the $z^{\prime}$ axis direction and the electric field gradient along the radial direction near the donor atom. When crystalline lattice and external electric field are absent, the wave function of this state is trans-formed into the hydrogen-like function, 
which corresponds to $1 \mathrm{~S}$ state. Shroedinger equation for this function in the electric field must be complemented by the perturbation operator $\Delta \hat{H}=e \cdot \varphi\left(\rho^{\prime}, z^{\prime}\right)\left(e=1.6 \cdot 10^{-19} \mathrm{C}\right.$ - elementary charge) [8]. Using (3), (4) in x, y, z basis (Fig. 1) we receive the following expression $\left(\rho^{2}=x^{2}+y^{2}\right)$ :

$$
\Delta \hat{H}=-\frac{2 V e}{\pi} \operatorname{arctg} \frac{a}{c}+e E_{c} \cdot z-\frac{e E_{c}^{\prime}}{2} \cdot z^{2}+\frac{e E_{c}^{\prime \prime}}{2} \cdot \rho^{2}=\Delta \hat{H}_{0}+\Delta \hat{H}_{1}+\Delta \hat{H}_{2}+\Delta \hat{H}_{3}
$$

The wave function $F_{j}(\vec{r})$ correction, specified by the perturbance (5), is:

$$
F_{j}=F_{j}^{(1 s)}+\Delta F_{j, 1}^{(1 s)}+\Delta F_{j, 2}^{(1 s)}
$$

where

$$
\Delta F_{j, 1}^{(1 s)}=\frac{\Delta H_{2 s, 1 s}}{\delta E} F_{j}^{(2 s)}
$$

- the first order correction of the perturbation theory, $\Delta H_{2 s, 1 s}=\int d^{3} r$. $\left(F_{j}^{(2 s)}(r)\right)^{*}(\Delta \widehat{H}) F_{j}^{(1 s)}(r)$ - matrix element of the perturbation operator and $\delta E=$ $E_{1 s}-E_{2 s}$ - energy residual of unperturbed donor states, and

$$
\Delta F_{j, 2}^{(1 s)}=-\frac{F_{j}^{(1 s)}}{2} \sum_{m \neq(1 s)} \frac{\left|\Delta H_{m, 1 s}\right|^{2}}{\left(E_{1 s}-E_{m}\right)^{2}}, m=2 p, 3 p, \ldots
$$

- the second order correction (here $E_{m}$ - unperturbed energy levels of donor, $\Delta H_{m, n}$ - matrix elements of the perturbation operator). During further calculations we shall use hydrogen-like functions as unperturbed wave functions. They are independent of $\mathrm{j}$ [10]:

$$
F^{(1 s)}(r)=\frac{1}{\sqrt{\pi} \cdot\left(a^{*}\right)^{3 / 2}} e^{-\frac{r}{a^{*}}} ; F^{(2 s)}(r)=\frac{1}{4 \sqrt{2 \pi} \cdot\left(a^{*}\right)^{3 / 2}}\left(2-\frac{r}{a^{*}}\right) e^{-\frac{r}{2 a^{*}}}
$$

where $r^{2}=x^{2}+y^{2}+z^{2}$ (Fig. 1), and $a^{*}=\frac{4 \pi \varepsilon \varepsilon_{0} \hbar^{2}}{m^{*} e^{2}}=20 \stackrel{0}{A}$ - effective Bohr radius [9], $\varepsilon=11.9, \varepsilon_{0}=8.85 \cdot 10^{-14} \mathrm{~F} / \mathrm{sm}$ - vacuum permittivity, $\hbar=6.62 \cdot 10^{-34} \mathrm{~J}^{*} \mathrm{sec}$, $m^{*}=0.31 m_{e}, m_{e}=9.1 \cdot 10^{-31} \mathrm{~kg}$. The second order terms in (6) give an expression a the respective correction of the hyperfine interaction constant of the donor atom:

$$
\frac{\Delta A(V)}{A} \equiv \frac{(F)^{2}-\left(F^{(1 s)}\right)^{2}}{\left(F^{(1 s)}\right)^{2}} \cong 2 \frac{\Delta F_{1}^{(1 s)}}{F^{(1 s)}}+2 \frac{\Delta F_{2}^{(1 s)}}{F^{(1 s)}}+\left(\frac{\Delta F_{1}^{(1 s)}}{F^{(1 s)}}\right)^{2}
$$


The second term in (10), according to 8.14, may be evaluated as:

$$
\frac{2 \Delta F_{2}^{(1 s)}}{F^{(1 s)}}=-\frac{9}{4} \cdot 4 \pi \varepsilon_{0}\left(a^{*}\right)^{3} \cdot \frac{E_{c}^{2}}{\Delta E}
$$

where $(\Delta E)^{-1}$ - some mean value of $\left(E_{m}-E_{1 s}\right)^{-1}$. At $\Delta E \sim 0.04 \mathrm{ev}, c=2 a=10$ $\mathrm{nm}$ it was obtained that $\left(\mathrm{V}\right.$ in volts) $\frac{2 \Delta F_{2}^{(1 s)}}{F^{(1 s)}}=-0.19 \mathrm{~V}^{2}$.

When calculating $\Delta H_{2 s, 1 s}$, we shall take into account that the item in the perturbance $\Delta \hat{H}_{0}$ does not contribute because of orthogonality of functions $F^{(1 s)}(\vec{r})$ and $F^{(2 s)}(\vec{r})$, and $\Delta \hat{H}_{1}$ does not contribute because of oddness of the function by $z$ under integral. Resulting,

$$
\Delta H_{2 s, 1 s}=\frac{2^{8} \sqrt{2}}{3^{6}} e E_{c}^{\prime}\left(a^{*}\right)^{2}-\frac{7^{2} 2^{5}}{3^{6} \sqrt{2}} e E_{c}^{\prime \prime}\left(a^{*}\right)^{2}
$$

Taking into account that $\frac{F^{(2 s)}}{F^{(1 s)}}=\frac{\sqrt{2}}{4},(10)$ appears as:

$$
\frac{\Delta A(V)}{A} \cong-9 \pi \varepsilon_{0}\left(a^{*}\right)^{3} \frac{E_{c}^{2}}{\Delta E}+\frac{2^{8} e E_{c}^{\prime}\left(a^{*}\right)^{2}}{3^{6} \delta E}\left(1-\frac{7^{2} E_{c}^{\prime \prime}}{2^{4} E_{c}^{\prime}}\right)+\left(\frac{2^{7}}{3^{6}} \frac{e E_{c}^{\prime}\left(a^{*}\right)^{2}}{\delta E}\left(1-\frac{7^{2} E_{c}^{\prime \prime}}{2^{4} E_{c}^{\prime}}\right)\right)^{2}
$$

The energy residual $\delta E$ can be evaluated, if we use the formula for hydrogen-like atoms $\delta E=-\frac{3}{8} \cdot \frac{e^{2}}{4 \pi \varepsilon \varepsilon_{0} a^{*}}$. The evaluation for silicon gives $\delta E=-0.023 \mathrm{ev}$. Using the abovementioned evaluations with the help of (13) for $c=2 a=10 \mathrm{~nm}$ we shall result:

$$
\frac{\Delta A(V)}{A} \cong 0.55 V-0.09 V^{2}
$$

Let us consider the case, when the A-gates have a form of infinitely long strips with the width $2 a=l_{A}$. They are placed at the distance D from the conducting substrate, from which the gate potential is counted out. Conducting similiar operations we receive for the donor atom HIC the following expression $\left(c=2 a=10 \mathrm{~nm}, \frac{D}{a}=100\right)$ :

$$
\frac{\Delta A(V)}{A} \cong-0.063 V^{2}
$$

Voltage in (15) is expressed in volts, as written above. 


\section{INFLUENCE OF THE TECHNOLOGICAL SPREAD OF DONOR ATOMS ON THE HIC. VOLTAGE ERROR ON A GATE}

Technological inaccuracies of the donor atom place under the gate relatively $x=0, z=c$ are designated $\delta x$ and $\delta z$. We investigate only the strip gate variant: Factorizing potential expression for strip gate by degrees of $\delta x$ and $\delta z$, we write it as:

$\varphi(x, z) \approx \varphi(0, c)+\frac{\partial \varphi(\delta x, c+\delta z)}{\partial z}(z-c)++\frac{1}{2 !} \frac{\partial^{2} \varphi(\delta x, c+\delta z)}{\partial z^{2}}(z-c)^{2}+\frac{\partial \varphi(\delta x, c+\delta z)}{\partial\left(x^{2}\right)} x^{2}$

where

$$
\begin{aligned}
& \frac{\partial \varphi(\delta x, c+\delta z)}{\partial z}=-\bar{E}_{c}\left\{1-\frac{\delta z}{c\left(1+a^{2} / c^{2}\right)}-\frac{(\delta x)^{2}\left(2 c^{2}-a^{2}\right)}{2\left(a^{2}+c^{2}\right)^{2}}\right\} ; \\
& \frac{\partial^{2} \varphi(\delta x, c+\delta z)}{\partial z^{2}}=\bar{E}_{c}^{\prime}\left\{1-\frac{\delta z\left(2 c^{2}-a^{2}\right)}{c\left(a^{2}+c^{2}\right)}-\frac{(\delta)^{2}\left(4 c^{4}+a^{2} c^{2}-a^{4}\right)}{2 c^{2}\left(a^{2}+c^{2}\right)^{2}}\right\} ; \\
& \frac{\partial \varphi(\delta x, c+\delta z)}{\partial\left(x^{2}\right)}=-\frac{1}{2} \bar{E}_{c}^{\prime \prime}\left\{1-\frac{\delta z\left(2 c^{2}-a^{2}\right)}{c\left(a^{2}+c^{2}\right)}-\frac{(\delta x)^{2}\left(2 c^{2}+a^{2}\right)}{2\left(a^{2}+c^{2}\right)^{2}}\right\} .
\end{aligned}
$$

We consider $\delta z \sim(\delta x)^{2}$, that is why corrections of $(\delta z)^{2}$ degree are omitted being of the next order. Using expressions (13), (15)-(17), we receive the final expression for the relative error of the HIC:

$$
\frac{\delta A}{A}=\delta z\left\{0.063 V^{2} \frac{2 c}{a^{2}+c^{2}}\right\}+(\delta x)^{2}\left\{0.063 V^{2} \frac{2 c^{2}-a^{2}}{\left(a^{2}+c^{2}\right)^{2}}-0.085 V \frac{2 c^{4}-a^{4}}{2 c^{2}\left(a^{2}+c^{2}\right)^{2}}\right\}
$$

The second item in (18) nullifies in a variety of values of $a, c$ and $V$. That is why by defining necessary relative error it is possible to determine structure parameters a and c and voltage $\mathrm{V}$, when the needed accuracy is realized. One percent relative error of the hyperfine interaction constant corresponds to the donor atom deviation along the $z$ axis, which is connected with the technological inaccuracy of placing in 2-3 $\mathrm{nm}$.

Voltage error on the gate, which is admissible during correctly conducted quantum calculation process, can be determined from the final expressions for HIC (14) and (15). The strip width of radio frequency pulses must be at least one order smaller than the resonant frequency of nuclear spins, which is about hundreds of $\mathrm{kHz}$. Considering the HIC value, 
which corresponds to the strip width of resonant pulse $\delta(\Delta A) \sim 10^{4} \mathrm{~Hz}$, for the gate voltage error we receive $10^{-4} \div 10^{-3} \mathrm{~V}$. It is worth of noting that in the case of round discs the distinguished voltage exists, that leads to the significant increasing of the admissible voltage error. It is caused by the presence of the linear by $\mathrm{V}$ term in (14).

\section{ELECTRON-NUCLEUS SPIN SYSTEM ENERGY SPECTRUM OF TWO INTERACTING DONOR ATOMS}

Consider two donor atoms separated from each other by distance l. This distance must satisfy the following condition: constant J of the electrons' effective exchange interaction, determined by the partial overlap of their wave functions, must correspond to the possibility of making two-qubit operations. Spin Hamiltonian of this system is:

$$
\begin{aligned}
& \hat{H}=2 \mu_{B} \vec{B}\left(\hat{\overrightarrow{S_{a}}}+\hat{\overrightarrow{S_{b}}}\right)+J\left(\hat{\overrightarrow{S_{a}}} \hat{\overrightarrow{S_{b}}}\right)+\Delta \hat{H}=\hat{H}_{0}+\Delta \hat{H}, \\
& \Delta \hat{H}=-g_{N} \mu_{N} \vec{B}\left(\hat{\overrightarrow{I_{a}}}+\hat{\overrightarrow{I_{b}}}\right)+A_{a}\left(\hat{\overrightarrow{I_{a}}} \hat{\overrightarrow{S_{a}}}\right)+A_{b}\left(\hat{\overrightarrow{I_{b}}} \hat{\overrightarrow{S_{b}}}\right)
\end{aligned}
$$

where $\hat{\overrightarrow{S_{a}}}, \hat{\overrightarrow{I_{a}}}, \hat{\overrightarrow{S_{b}}}, \hat{\overrightarrow{I_{b}}}$ - spin operators of the electron and the nuclei for the first and the second atom, $\vec{B}$ - the magnetic field (it is directed in parallel to $z$ axis (see Fig. 1)), $A_{a}$ and $A_{b}$ - hyperfine interaction constants depending, generally speaking, on gate potentials, $\mu_{B}$ - Bohr magneton, $\mu_{N}$ - nuclear magneton, $g_{N}=2.26$ - Lande factor for ${ }^{31} \mathrm{P}$.

We consider $\left|M_{a} ; M_{b} ; m_{a} ; m_{b}\right\rangle$ to be the basis (eigen states in the strongest fields), where $M_{a}, M_{b}, m_{a}, m_{b}$ - quantum numbers of the electrons' and nuclei's spin projections. Designating them by arrows up $(\uparrow)$ and down $(\downarrow)$, we have (numbers are strings or rows in the matrix):

$$
\begin{array}{llll}
|1\rangle=|\uparrow \uparrow \uparrow \uparrow\rangle & |5\rangle=|\uparrow \downarrow \uparrow \uparrow\rangle & |9\rangle=|\downarrow \uparrow \uparrow \uparrow\rangle & |13\rangle=|\downarrow \downarrow \uparrow \uparrow\rangle \\
|2\rangle=|\uparrow \uparrow \uparrow \downarrow\rangle & |6\rangle=|\uparrow \downarrow \uparrow \downarrow\rangle & |10\rangle=|\downarrow \uparrow \uparrow \downarrow\rangle & |14\rangle=|\downarrow \downarrow \uparrow \downarrow\rangle \\
|3\rangle=|\uparrow \uparrow \downarrow \uparrow\rangle & |7\rangle=|\uparrow \downarrow \downarrow \uparrow\rangle & |11\rangle=|\downarrow \uparrow \downarrow \uparrow\rangle & |15\rangle=|\downarrow \downarrow \downarrow \uparrow\rangle \\
|4\rangle=|\uparrow \uparrow \downarrow \downarrow\rangle & |8\rangle=|\uparrow \downarrow \downarrow \downarrow\rangle & |12\rangle=|\downarrow \uparrow \downarrow \downarrow\rangle & |16\rangle=|\downarrow \downarrow \downarrow \downarrow\rangle
\end{array}
$$


When nuclear spins are absent $(\Delta \hat{H}=0)$, Hamiltonian $\frac{\hat{H}_{0}}{J}$ has four times four degenerated eigen states (Fig. 2). Two underlying electron levels have the point of crossing $\mathrm{C}$ when $\beta=1$.

Symmetric matrix16*16, corresponding to the matrix of eigen states of Hamiltonian $\Delta \hat{h} \equiv \frac{\Delta \hat{H}}{J}$, in the considered basis has only diagonal elements $-\mu+\frac{\alpha_{a}+\alpha_{b}}{4}, \frac{\alpha_{a}-\alpha_{b}}{4},-\frac{\alpha_{a}-\alpha_{b}}{4}$, $\mu-\frac{\alpha_{a}+\alpha_{b}}{4},-\mu+\frac{\alpha_{a}-\alpha_{b}}{4}, \frac{\alpha_{a}+\alpha_{b}}{4},-\frac{\alpha_{a}+\alpha_{b}}{4}, \mu-\frac{\alpha_{a}-\alpha_{b}}{4},-\mu-\frac{\alpha_{a}-\alpha_{b}}{4},-\frac{\alpha_{a}+\alpha_{b}}{4}, \frac{\alpha_{a}+\alpha_{b}}{4}, \mu+\frac{\alpha_{a}-\alpha_{b}}{4}$, $-\mu-\frac{\alpha_{a}+\alpha_{b}}{4},-\frac{\alpha_{a}-\alpha_{b}}{4}, \frac{\alpha_{a}-\alpha_{b}}{4}, \mu+\frac{\alpha_{a}+\alpha_{b}}{4}$, where $\mu \equiv \frac{g_{N} \mu_{N} B}{J}, \alpha_{a} \equiv \frac{A_{a}}{J}, \alpha_{b} \equiv \frac{A_{b}}{J}$ and non-diagonal elements, from which we point out only up-diagonal elements:

$$
\begin{aligned}
& (\Delta \hat{h})_{5,2}=(\Delta \hat{h})_{7,4}=(\Delta \hat{h})_{13,10}=(\Delta \hat{h})_{15,12}=\frac{\alpha_{b}}{2} \\
& (\Delta \hat{h})_{9,3}=(\Delta \hat{h})_{10,4}=(\Delta \hat{h})_{13,7}=(\Delta \hat{h})_{14,8}=\frac{\alpha_{a}}{2}
\end{aligned}
$$

Since the projection of full electron and nuclear spin in the direction of the magnetic field $M+m=M_{a}+M_{b}+m_{a}+m_{b}$ is conserved, the matrix16*16 breaks down into 5 reduced matrixes of smaller dimension, corresponding to the values $M+m=0, \pm 1, \pm 2$, which diagonalize independently. The next states correspond to these 5 matrixes:

$$
\begin{aligned}
& m+M=0:|4\rangle,|6\rangle,|7\rangle,|10\rangle,|11\rangle,|13\rangle \\
& m+M=1:|2\rangle,|3\rangle,|5\rangle,|9\rangle \\
& m+M=-1:|8\rangle,|12\rangle,|14\rangle,|15\rangle \\
& m+M=2:|1\rangle \\
& m+M=-2:|16\rangle .
\end{aligned}
$$

With the help of the found matrix, introducing Hamiltonian (19), the picture of the hyperfine splitting of two donor atoms' electrons' levels was achieved. It is shown for $\alpha_{a}=$ $0.3 ; \quad \alpha_{b}=0.4$ in figure 3 .

The interaction of electronic and nuclear spins not only takes down the four times degeneracy, but brings near point $\mathrm{C}$ the phenomenon of anticrossing of the levels, which have the same value of $m+M$.

For two crossing electronic levels it takes place for the states with $m+M=-1 \mid 15>$ and $\mid 12>$ (two solid bold lines) and with $m+M=0 \mid 10>$ and $\mid 13>$ (two dashed lines) 
(see Fig. 3).

As the result of this anticrossing the state $\mid 15>$ with $M_{a}+M_{b}=-1$ and $m_{a}+m_{b}=0$, when $\mathrm{J}$ increases (with the help of J-gate), makes a transition to the $\mid 12>$ state with $m_{a}+m_{b}=-1$ and $M_{a}+M_{b}=0$, i.e. the simultaneous inversion of one nuclear spin and one electron spin takes place (the information about nuclear spin condition is transfered to the electron spin). Herewith the electron triplet state $(S=1)$ makes a transition to the singlet one $(S=0)$.

The energy residual of the underlying anticrossing state $\mid 15>$ and crossing state $\mid 14>$ in the second order of perturbation theory for the strong magnetic fields $(\beta>>1)$ and for $A_{a}=A_{b} \equiv A$, according to [5], is

$$
E_{14}-E_{15}=2 \pi \hbar v_{J}=\frac{(A / 2)^{2}}{2 \mu_{B} B-J}-\frac{(A / 2)^{2}}{2 \mu_{B} B},
$$

where $v_{J}$ — transition frequency, which corresponds to projection changing of two nuclear spins. It increases, when $\beta$ decreases.

In [5| the levels $\mid 15>$ and $\mid 14>$ are suggested to be used as "working" ones to conduct the calculation process and to measure the final state.

However, we also can propose an alternative variant. It is possible to use the second pair of anticrossing levels with $m+M=0$. Underlying state of the anticrossing level $\mid 13>$ in the strong fields corresponds to $M_{a}+M_{b}=-1, m_{a}+m_{b}=1$, and above-lying state $\mid 14>$ corresponds to $M_{a}+M_{b}=-1, m_{a}+m_{b}=0$, i.e. they differ by the inverted value of one of the nuclear spins.

When $\mathrm{J}$ increases, the state $\mid 13>$, moving through the anticrossing point, makes a transition to the state $\mid 10>$ with $M_{a}+M_{b}=0$ and $m_{a}+m_{b}=0$, i.e. the simultaneous inversion of the electron spin such as the nuclear one happens (the information about nuclear spin is transferred to the electron spin, too).

According to [5], the measurement process of nuclear spin is s upposed to be implemented in two stages. By changing the value of the electron exchange interaction $\mathrm{J}$ from $J<2 \mu_{B} B$ up to $J>2 \mu_{B} B$ the adiabatic transition of the nuclear spin condition is made from triplet 
electron ground state to the singlet one. Then the electron state measurement is made.

If at first the electrons of two donors are at the triplet state, simultaneously being placed on its own atom, then, when J increases, it is more energy-advantageous for the both electrons to exist in the singlet state being placed together on the one of the donors, if the bonding energy of two electrons on the single donor exceeds the bonding energy for each electron on its own donor $\left(D^{-}\right.$-state). As a result the charge carrying takes place from one donor to another. As it is pointed out in [5], this fact can be registered by highly sensitive single-electron capacity methods.

At the present time different methods of the spin states measurement are discussed. In 11] it is suggested to use a scheme, agreeably called "turnstile", which omits current of electrons only with the definite spin state. This electric method allows to measure electron spin states. Another approach was discussed in [12]. It is based on using magnetic resonant force microscopy.

\section{CONCLUSION}

In this work an influence of the electric field, inducing by the A-gate (for the gate as a round disc and as a strap) on the hyperfine interaction constant was analyzed in details. The weaker dependence of the hyperfine interaction constant from the depth of underlying donors under the gate, and also decreasing of its magnitude when the distance from the gate to the substrate increases are noted. Conducted calculations with two gate types allows us to make a conclusion about significant dependence of the hyperfine interaction constant from the form of a gate.

Parameters a and c (5-10 nm) of the structure at which errors rate can be minimized are determined by (17). Obtained expressions allow us to determine admissible values of technological inaccuracies $\delta x$ and $\delta z(2-3 \mathrm{~nm})$ when the constant A errors and working potential values $\mathrm{V}$ (0.1-1 volts) of a gate are defined. In work the full energy spectrum of the electron-nuclear spin system of two interacting donor atoms for $A_{a} \neq A_{b}$ was calculated. The 
existence of two pairs of anticrossing levels at the ground electron state, corresponding to the full electron and nuclear spin projection $m_{a}+m_{b}+M_{a}+M_{b}=-1$ and $m_{a}+m_{b}+M_{a}+M_{b}=0$ was demonstrated. Underlying components of these pairs correspond to the transition from the triplet electron state $(S=1)$ to the singlet one $(S=0)$, when the field decreases. If the full electron spin projection changes from $M_{a}+M_{b}=-1$ to $M_{a}+M_{b}=0$, the nuclear spin projection also correspondingly changes. Thus the information about nuclear spin state transfers to the electron system. Both pairs of anticrossing levels may be used to conduct calculation and measurement process. Herewith we must use the frequency, determined by splitting between underlying anticrossing levels $(\mid 13>$ or $\mid 15>)$ and a bove-lying level $\mid 14>$, to change nuclear spin state.

In conclusion authors would like to thank L.A. Openov for the valuable comments.

[1] Gershenfeld N.A., Chuang I.L., Bulk Spin-Resonance Quantum Computation. Science, 1997, v.275, 17 Jan, pp.350-356.

[2] Cory D.G., Price M.D., Havel T.F. Nuclear Magnetic Resonance Spectroscopy: An Experimentally Accessible Paradigm for Quantum Computing. // Physica D, 1997, v.120, N.1-2, pp. $82-101$.

[3] Chuang I.L., Gershenfeld N., Kubinec M.G., Leung D.W. Bulk Quantum Computation with Nuclear Magnetic Resonance: Theory and Experiment. // Proc. Roy. Soc. Lond., 1998, v.A454, N.1969, pp. 447-467.

[4] Cory D.G., Fahmy A.M., Havel T.F. Ensemble Quantum Computing by NMR Spectroscopy. // Proc. Natl. Acad. Sci. USA, 1997, v.94, N.5, pp.1634-1639.

[5] Kane B.E. A silicon-based Nuclear Spin Quantum Computer. // Nature, 1998 v.393, 14 May, pp. 133-137. 
[6] Ludwig J., Wudberi G. Electronniy spinoviy rezonans v poluprovodnikakh. / Perevod s angl. Podd red. N. A. Penina. - M., Mir, 1964, p.148.

[7] Landau L.D., Lifshits E.M. Elektrodinamika sploshnikh sred. - M.: Nauka, 1992, pp.38-51.

[8] Valiev K.A., Kokin A.A. Poluprovodnikoviye YaMR kvantoviye kompjuteri s individualnim obrascheniem k kubitam. // Mikroelektronika, 1999, v.28, N.5, pp.326-337.

[9] Kohn W. Shallow Impurity States in Silicon and Germanium. // Solid State Physics / Eds. F.Seitz, D.Turnbull. - N.Y.- Lond.: Acad. Press., 1957, v.5, pp.257-320.

[10] Shiff L. Kvantovaya mekhanika. / Perevod s angl. G.A. Zaytseva. — Moskva, 1959, p.106.

[11] Molotkov S.N., Nazin S.S. Izmereniya sostoyaniya otdelnogo spina pri pomoschi "turniketa". // Pisma v ZhETF, 1999, v.70, pp.14.

[12] Berman G.P., Doolen G.D., Hammel P.C., Tsifrinovich V.I. Solid-State Nuclear Spin Quantum Computer Based on Magnetic Resonance Force Microscopy. //Phys. Rev. B, v.61, p.14694 (2000).

[13] Berman G.P., Campbell D.K., Doolen G.D., Nagaev K.E. Dynamics of the Measurement of Nuclear Spins in a Solid-State Quantum Computer. // 1999, E-print LANL, condmat/9905200.

[14] Valiev K.A., Kokin A.A. Solid-State NMR Quantum Computer with Individual Access to Qubits and some their Ensemble Developments. // 1999, E-print LANL quant-ph/9909008. 


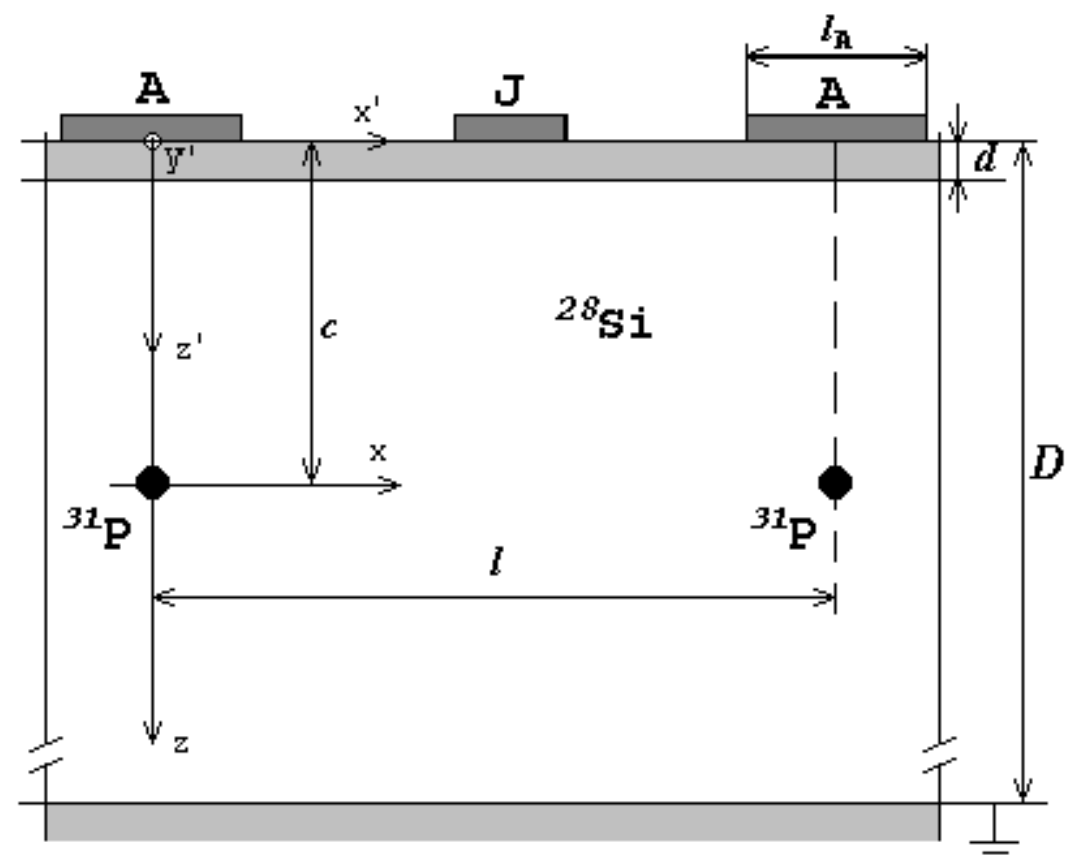




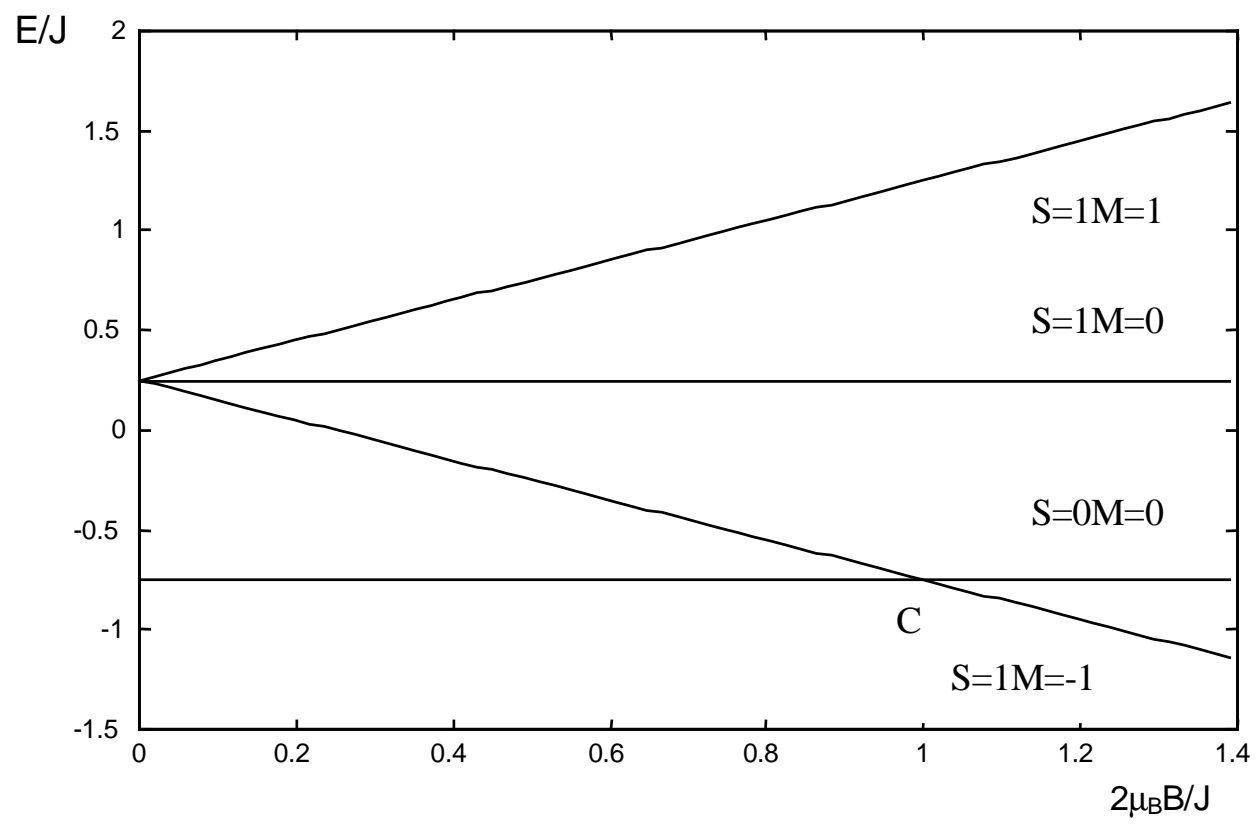




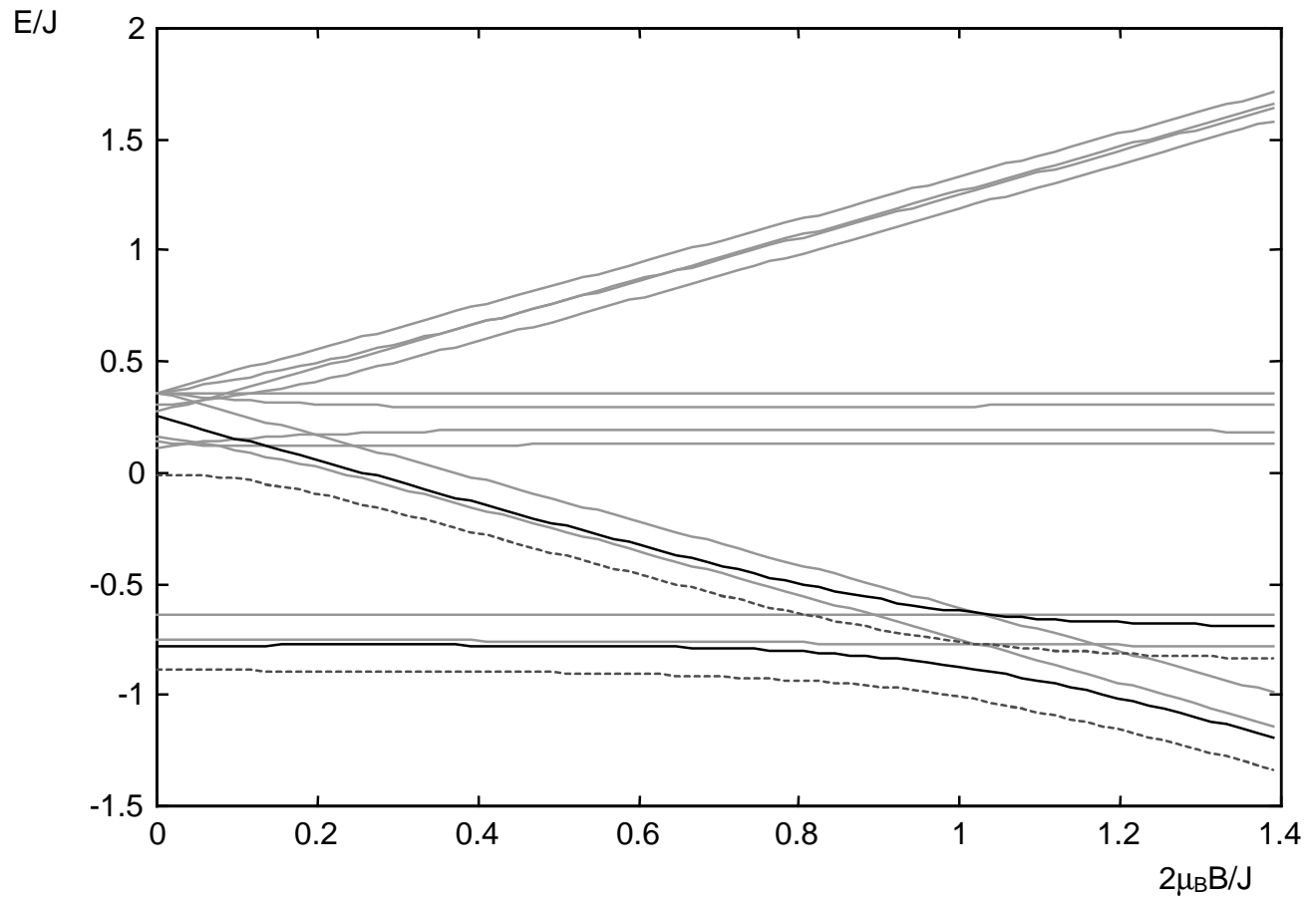

